\title{
CAPITULO 51
}

FARMACOCINÉTICA: ABSORÇÃO E VIAS DE ADMINISTRAÇÃO

DOI 10.4322/978-65-995353-2-1.c51

Carolina Dourado de Faria ${ }^{1}$, Fernando Antônio Ramos Schramm Neto ${ }^{2}$, Yuri de Jesus Machado $^{3}$, Lucas Furlan Cirqueira de Souza ${ }^{4}$, Isabelly Raiane Silva dos Santos ${ }^{5}$, Carolina Woinarovicz Meneghetti ${ }^{6}$, Liara de Oliveira Teixeira ${ }^{7}$, Demerval de Pinho Borges Netto ${ }^{8}$, Antônio Lucas Farias da Silva ${ }^{9}$, Geísa de Morais Santana ${ }^{10}$.

\author{
${ }^{1}$ Universidade Salvador, carolinain11@gmail.com; \\ ${ }^{2}$ Universidade Salvador, fernando78541@ @otmail.com; \\ ${ }^{3}$ Universidade Salvador, mjyuri1999@gmail.com; \\ ${ }^{4}$ Universidade de Uberaba, lucasfurlan7@ hotmail.com; \\ ${ }^{5}$ Universidade Federal do Pará, isabelly.santos@ifpa.edu.br; \\ ${ }^{6}$ Universidade Estadual de Ponta Grossa, carolinawmeneghetti@gmail.com; \\ ${ }^{7}$ Universidade Federal de Santa Maria, liarateixeira6@gmail.com; \\ ${ }^{8}$ Centro Universitário Unifacid, demervalpinho15@outlook.com; \\ ${ }^{9}$ Centro Universitário Unifacid, lucas1992farias@gmail.com; \\ ${ }^{10}$ Universidade Estadual do Piauí, geisasantana97@gmail.com.
}

\section{RESUMO}

Objetivo: Discutir sobre as principais evidências científicas acerca do processo de absorção e vias de administração envolvendo a farmacocinética. Método: Foi realizado uma revisão narrativa de literatura. As bases de dados bibliográficas utilizadas foram PubMed, SciELO e LILACS. Os seguintes descritores foram usados na pesquisa: "Pharmacokinetics", "Absorption", "Drug administration routes". Resultados: A absorção constitui-se como o primeiro processo da farmacinética, isto é, do caminho que o medicamento percorre após a administração no corpo humano. É nesta etapa que irá ocorrer a passagem das substâncias medicamentosas para a corrente sanguínea para, posteriormente, ser distribuída ao meio intracelular. Os princípios farmacocinéticos dependem da permeabilidade do fármaco, que, por sua vez, dependem de uma série de características, tais como: lipossolubilidade, gradiente de concentração, via de administração, ionização, dentre outras. Considerações Finais: $O$ entendimento acerca da etapa de absorção de fármacos, a biodisponibilidade e vias de 
administração é de fundamental importância para minimizar os riscos de efeitos indesejados no paciente.

Palavras-chave: Farmacologia; Compostos Orgânicos; Atenção à Saúde.

Área Temática: Ciências da Saúde.

E-mail do autor principal: carolinain11@gmail.com

\section{INTRODUÇÃO}

Para que exista um melhor entendimento a respeito da ação terapêutica dos fármacos, se faz necessário compreender a farmacocinética (Brunton, 2012), isto é, a forma com que o organismo processa os medicamentos (Pereira, D. G; 2007): a absorção, a distribuição, o metabolismo e a excreção dos fármacos (ADME) (Davis, C. \& Harris, S. R, 2017). Em outras palavras, é importante compreender o aproveitamento de determinada substância pelo organismo (biodisponibilidade) e o tempo gasto no seu local de ação, de maneira a assegurar um bom desfecho terapêutico ao paciente, e minimizando a ocorrência de efeitos indesejados (Brunton, 2012; Pereira, D. G, 2007).

Os princípios farmacocinéticos (ADME) dependem da permeabilidade do fármaco através das membranas celulares, e, portanto, das características físico-químicas das suas moléculas. Alguns fatores decisivos no transporte e consequente biodisponibilidade do fármaco são: lipossolubilidade, gradiente de concentração, maior área de contato e vascularização, e ionização (Brunton, 2012; Davis, C. \& Harris, S. R, 2017).

A permeabilidade do fármaco depende, na maioria das vezes, das seguintes características: Lipossolubilidade, gradiente de concentração, área de superfície e vascularização, além da ionização. Lipossolubilidade se trata da capacidade do fármaco de se difundir através das bicamadas fosfolipídicas que compõem as membranas das células. Pode ser medida por meio do coeficiente de partição óleo-água. Quanto maior for o coeficiente de partição, maior a lipossolubilidade. Quanto ao gradiente de concentração, fármacos livres, não ionizadas, podem se difundir de um local mais concentrado, para outro, menos concentrado. Área de superfície e vascularização também possui importante relação com a permeabilidade, visto que, quanto maior a área de contato do órgão com as moléculas do fármaco e quanto maior a vascularização de determinado local, melhor será a absorção do fármaco para a circulação sanguínea. Por fim, a grande maioria dos fármacos são ácidos fracos ou bases fracas, podendo existir na forma não ionizada ou molecular (capaz de atravessar as membranas celulares, e, portanto, mais bem absorvido) ou na forma ionizada (mais bem excretado por ser 
hidrossolúvel). Para saber quanto de cada forma irá existir, dependerá do gradiente de $\mathrm{pH}$ através da membrana e do pKa processo absortivo (Davis, C. \& Harris, S. R, 2017).

Fundamentando-se nisso, o objetivo deste trabalho é discutir sobre as principais evidências científicas acerca do processo de absorção e vias de administração envolvendo a farmacocinética.

\section{MÉTODO}

O presente estudo constitui uma revisão narrativa da literatura sobre a etapa de absorção na farmacocinética e vias de administração. Os dados foram obtidos por meio de levantamento bibliográfico entre abril e maio de 2021 e a revisão foi conduzida entre maio e junho de 2021 . Como critério de inclusão, fez-se seleção de publicações dos últimos 15 anos, nos idiomas inglês e português. Os descritores em inglês utilizados para o cruzamento foram: "Pharmacokinetics”, “Absorption”, "Drug Administration routes”. O operador booleano “AND" foi usado para auxiliar nas pesquisas. As bases de dados eletrônicas utilizadas para a pesquisa foram PubMed, SciELO e LILACS, bem como livros publicados sobre o tema. É importante destacar que o estudo se pautou nas características de uma revisão narrativa. Dessa forma, não foi desenvolvido com base em um questionamento específico, de forma sistematizada, mas sim em tema amplo com seleção de informações em publicações gerais sobre o assunto para atualizar o conhecimento do leitor acerca desta temática.

\section{RESULTADOS E DISCUSSÃO}

O pKa se refere ao pH no qual a metade do fármaco, seja ele ácido ou básico, está dissociado. Quando ácidos se dissociam, normalmente, ocorre formação de íons $\left(\mathrm{HA} \rightarrow \mathrm{H}^{+}+\right.$ A), e quando bases se dissociam, normalmente, não ocorre formação de partícula ionizada $\left(\mathrm{BH}^{+}\right.$ $\rightarrow \mathrm{H}^{+}+\mathrm{B}$ ). Quando o fármaco, seja ele ácido ou básico, estiver em um meio no qual o $\mathrm{pH}$ for menor que seu pKa, irá predominar sua forma protonada, já que o ambiente está repleto de $\mathrm{H}^{+}$. A forma protonada do fármaco ácido condiz com sua forma não ionizada (HA), enquanto a forma protonada da base $\left(\mathrm{BH}^{+}\right)$condiz com sua forma ionizada.

Já quando o fármaco estiver em um meio com pH maior que seu, irá ocorrer o inverso, havendo predomínio da forma não protonada. A forma não protonada do ácido condiz com sua forma ionizada $\left(\mathrm{H}^{+}+\mathrm{A}^{-}\right)$, enquanto a base não protonada condiz com sua forma não ionizada $\left(B+\mathrm{H}^{+}\right.$) (Brunton, 2012; Davis, C. \& Harris, S. R, 2017).

Uma correlação clínica que pode ser feita para facilitar o entendimento é quanto à metabolização da lactulose, açúcar utilizado no tratamento da encefalopatia hepática, patologia 
que, dentre outras consequências, causa hiperamonemia. A microbiota intestinal é capaz de transformar a lactulose em ácido lático, acidificando, dessa forma, as massas fecais. A redução de $\mathrm{pH}$ faz com que a amônia $\left(\mathrm{NH}_{3}\right)$ se transforme em amônio $\left(\mathrm{NH}_{4}{ }^{+}\right)$, sua forma ionizada, e, portanto, passível de excreção renal (Davis, C. \& Harris, S. R, 2017).

Algumas observações importantes a se ter em mente para dar continuidade ao tema são:

A) Somente moléculas desconectadas de proteínas plasmáticas, sejam elas ionizadas ou não ionizadas, podem ser filtradas (Davis, C. \& Harris, S. R, 2017);

B) As moléculas não ionizadas são aquelas passíveis de serem reabsorvidas ou secretadas, tendo em vista que, estes processos requerem a passagem através de membranas celulares, enquanto os fármacos ionizados não conseguem realizar esta passagem, e ficam, portanto, retidos no filtrado para posterior excreção (Davis, C. \& Harris, S. R, 2017).

Este último ponto é de extrema relevância para a clínica, uma vez que a manipulação do pH da urina pode influenciar na eliminação de um agente tóxico, por exemplo. A acidificação da urina irá aumentar o processo de ionização das bases fracas e a alcalinização da urina irá aumentar a ionização de ácidos fracos, impedindo a reabsorção, possibilitando a excreção forçada destes compostos (Davis, C. \& Harris, S. R, 2017; Zucoloto, A.D et al,2017).

Com tudo isso, dá para chegarmos em uma regra prática: se a intenção for de que o fármaco permaneça no organismo de um indivíduo, ela deve ser colocada em um ambiente semelhante, visto que irá predominar sua forma não ionizada. Em outras palavras, um fármaco de caráter ácido é melhor absorvido em meio ácido e um fármaco básico é melhor absorvido em meio básico. Se a intenção for de eliminar o fármaco, no entanto, deve-se colocá-lo em um ambiente de caráter oposto, já que irá predominar sua forma ionizada. Um fármaco de caráter ácido é mais bem excretado em meio básico e um fármaco básico é mais bem excretado em meio ácido (Brunton, 2012; Davis, C. \& Harris, S. R, 2017).

\subsection{ABSORÇÃO E ASPECTOS CLÍNICOS}

A absorção corresponde ao processo de passagem do fármaco do seu sítio de administração para a circulação sanguínea (Brunton, 2012; Davis, C. \& Harris, S. R, 2017; Souza et al, 2007; Katzung, 2017). A permeabilidade tem papel intrínseco na transferência de determinada substância de um compartimento (local de administração) a outro (sangue) à medida que possibilita o transporte por meio de membranas biológicas (Brunton, 2012; Davis, C. \& Harris, S. R, 2017; Souza et al, 2007).

A absorção está intimamente relacionada com a biodisponibilidade. Este termo se refere

E - book Tripé do Ensino Superior: Ensino, Pesquisa e Extensão 
à porcentagem inalterada da dose, administrada por qualquer via, que consegue efetivamente chegar na circulação sistêmica e, por conseguinte, no seu local de ação (Brunton, 2012; Davis, C. \& Harris, S. R, 2017; Katzung, 2017). Diversos fatores podem afetar a absorção de substâncias, como elementos fisiológicos relacionados ao trato gastrointestinal, características físico-químicas da droga, a forma farmacêutica administrada, entre outros.

A biodisponibilidade de um fármaco pode ser influenciada durante o período pósprandial pelos elementos fisiológicos do TGI, com destaque para a redução da velocidade de esvaziamento gástrico, aumento do trânsito intestinal e competição pelos sítios de absorção. A presença do alimento também pode modificar o $\mathrm{pH}$ do conteúdo gastrointestinal, de modo a facilitar ou dificultar a desintegração de determinada cápsula, ou interferir na ionização de certo fármaco, promovendo alterações na velocidade e extensão de absorção (Souza et al, 2007; Rossato, J.P.M, 2015).

Outro fator que pode contribuir para a redução da biodisponibilidade é o metabolismo de primeira passagem, que, em resumo, é um fenômeno no qual a concentração do fármaco é significativamente reduzida pelo fígado antes de atingir a circulação sistêmica (Brunton, 2012), mas isso será discutido em outro capítulo, quando formos falar de metabolismo dos fármacos.

A administração medicamentosa, de uma forma branda, pode ser dividida em intravascular e extravascular. A aplicação que ocorre diretamente no interior dos vasos permite que haja uma biodisponibilidade total do fármaco, visto que não envolve o processo de absorção. Por outro lado, a dose de um fármaco administrada por via extravascular (oral, subcutânea, intramuscular, inalatória) provavelmente não chegará intacta à circulação sistêmica por intercorrências diversas no processo absortivo (Brunton, 2012; Davis, C. \& Harris, S. R, 2017; Katzung, 2017).

\subsection{VIAS DE ADMINISTRAÇÃO}

Existem diversas vias pelas quais a terapêutica medicamentosa de um paciente pode ser realizada. É preciso, no entanto, saber qual a opção mais qualificada para cada caso. Para isso, é necessário haver entendimento quanto às vantagens e desvantagens das diferentes formas de aplicação medicamentosa.

\subsubsection{VIAS ENTERAIS}

\section{Via oral}

A administração de medicamentos por via oral é a mais comum, segura e econômica, além de cômoda para a maior parte dos indivíduos, tendo em vista a ausência de dor e 
possibilidade de automedicação. Estes medicamentos podem ter formulações sólidas (comprimidos, cápsulas, drágeas) ou líquidas (elixires, xaropes) (Brunton, 2012; TRIPATHI, KD, 2006; Le, J, 2019).

Existem alguns fatores que podem limitar a absorção destes fármacos, como: o tempo de dissolução do medicamento nas secreções gastrointestinais;

I- o tempo de esvaziamento gástrico;

II- o pH do meio: como a maior parte da absorção do fármaco pelo trato gastrointestinal ocorre por difusão simples, ela será facilitada quando o fármaco estiver em sua forma mais permeável, não ionizada;

III- efeito de primeira passagem: os fármacos presentes no trato gastrointestinal podem ser metabolizados por enzimas da flora ou mucosa intestinais, ou do fígado, antes de alcançarem a circulação sistêmica.

Como a absorção da maioria destes fármacos ocorrem no estômago e intestino, podem ocorrer irritações nestes órgãos. Anti-inflamatórios, por exemplo, podem gerar úlceras gástricas. Outra desvantagem é relacionada à perda de uma porcentagem do fármaco pelo metabolismo de primeira passagem (Brunton, 2012).

A via sublingual propicia uma rápida absorção, diretamente para a circulação sanguínea, devido à vasta vascularização e à baixa espessura da mucosa sublingual. A ação do fármaco pode ocorrer em poucos minutos. Apenas medicamentos lipossolúveis e não irritantes podem ser administrados por essa via. A principal vantagem é evitar a passagem pelo fígado, visto que a drenagem da boca vai em direção à veia cava superior, desviando da circulação porta, poupando alterações na biodisponibilidade (Brunton, 2012; TRIPATHI, KD, 2006).

Às vezes, o princípio ativo do fármaco é colocado dentro de micelas de lipossoma, e este complexo, ao chegar no estômago ou intestino, começa a liberar o princípio ativo de forma lenta. Podemos, dessa forma, colocar uma dosagem bem alta dentro do lipossoma, que terá duração prolongada, sem necessidade de ingestão de outra dose. A dose farmacológica será muito grande porque a liberação é lenta. Essas são as chamadas preparações de liberação controlada (Brunton, 2012).

\section{Via retal}

A via retal pode ser utilizada como alternativa da via oral para crianças, para pacientes inconscientes, indivíduos com transtornos psicológicos e para pacientes que apresentam vômitos e náuseas. Esta forma de administração também pode ser útil para substituir certas medicações que provocam muita irritação gastrointestinal ou elevada perda por metabolismo

E - book Tripé do Ensino Superior: Ensino, Pesquisa e Extensão 
hepático de primeira passagem (LIMA, M. F, 2008). Apenas cerca da metade do composto absorvido pelo reto passará pelo fígado, sendo reduzida a perda farmacológica por metabolismo de primeira passagem, quando comparada à via oral (Brunton, 2012). Existem, no entanto, algumas desvantagens que precisam ser consideradas, como a absorção irregular e incompleta do fármaco, além de possibilidade de irritação da mucosa retal (Brunton, 2012).

\subsubsection{VIAS PARENTERAIS}

As vias parenterais estão relacionadas à aplicação do fármaco por injeção, liberando-a diretamente no líquido tecidual ou no sangue, sem precisar atravessar a mucosa intestinal. Ocorre a liberação da forma ativa do fármaco, promovendo uma ação quase que imediata. Por esse motivo, a dose eficaz pode ser administrada com maior precisão. Esta via faz-se de grande importância quando o paciente está inconsciente e incapaz de colaborar com a deglutição de um medicamento por via oral, por exemplo (Brunton, 2012; TRIPATHI, KD, 2006; Le, J, 2019). É preciso ficar atento, todavia, a algumas desvantagens: a assepsia deve ser mantida e a técnica utilizada deve ser a correta para evitar acidentes, como sepse, infecção, hematoma, transfixação, entre outros. Além disso, as injeções podem ser doloridas e dificultosas para autoaplicações (Brunton, 2012; TRIPATHI, KD, 2006).

\section{Intravenosa (IV)}

A via intravenosa permite um efeito rápido, biodisponibilidade de $100 \%$ e controle máximo sobre a quantidade de fármaco administrada. Além disso, a liberação farmacológica pode ser mais bem controlada e precisa. A indução de anestesia operatória e administração de soluções irritantes são alguns exemplos de quando esta via precisa ser utilizada. Os fármacos são administrados lentamente para que a dose seja distribuída amplamente na corrente sanguínea e para que seja ajustada de acordo com a resposta do paciente (Brunton, 2012).

Após a administração intravenosa dos fármacos, é preciso que o paciente seja monitorado atentamente, pois os medicamentos podem atingir altas concentrações plasmáticas em pouco tempo, gerando reações indesejáveis. Além do mais, após a injeção da substância, geralmente, não é possível retirá-la da circulação (Brunton, 2012). O tipo de medicamento administrado por essa via não pode ser dissolvido em veículos oleosos, conter compostos que se precipitam no sangue ou que lisem eritrócitos. Ademais, o paciente geralmente precisa da ajuda profissional para a realização desse procedimento, por ser doloroso e requerer certa cautela (Brunton, 2012).

\section{Intramuscular (IM)}

E - book Tripé do Ensino Superior: Ensino, Pesquisa e Extensão 
A via de administração intramuscular deposita a medicação profundamente em músculos estriados esqueléticos, como o deltoide, tríceps, glúteo máximo etc., possibilitando, na maioria das vezes, a rápida e completa absorção do fármaco, tendo em vista que os músculos costumam ser bastante vascularizados (TRIPATHI, KD, 2006). A absorção do fármaco pode ser limitada. No entanto, em alguns casos, devido às suas propriedades físico-químicas, ou por fatores fisiológicos, como a perfusão sanguínea no local de aplicação (LIMA, M. F, 2008).

Os fármacos administrados por esta via podem estar em soluções aquosas ou em preparações de depósito, sendo absorvidas, respectivamente, de forma rápida ou lenta (LIMA, M. F, 2008).

\section{Subcutânea (SC)}

A administração medicamentosa por via subcutânea possui, em geral, uma absorção mais lenta e efeito prolongado, já que o tecido subcutâneo é pouco vascularizado. Todavia, o tempo de absorção pode ser alterado de forma proposital. Por exemplo, o acréscimo de um agente vasoconstritor na solução a ser injetada pode retardar esse processo (Brunton, 2012). É preciso ter cautela quanto ao fármaco que será administrado por essa via, pois fármacos que irritam os tecidos, além de provocarem dor intensa no paciente, em função do suprimento de nervos no tecido subcutâneo, podem gerar necrose e descamação tecidual (TRIPATHI, KD, 2006; LIMA, M. F, 2008).

\subsection{OUTRAS VIAS: TRANSDÉRMICA, TÓPICA E RESPIRATÓRIA}

A pele intacta não é permeável a fármacos, no entanto, a exposição da derme, em situações de queimadura ou inflamação, por exemplo, com consequente aumento do fluxo sanguíneo local, possibilita que solutos se tornem permeáveis e que ocorra absorção sistêmica. A absorção pela pele pode ser aumentada pela suspensão da substância em um veículo oleoso (lipossolúvel) e pela fricção deste produto no local da aplicação (Brunton, 2012).

As aplicações tópicas visam a obtenção de uma ação localizada sobre a região afetada. Os princípios ativos passam, em quantidade mínima, para a circulação sanguínea para evitar os efeitos no resto do organismo. Nesta via, estão inclusas as seguintes administrações: cutânea (pomada, gel, cremes, solução em pó), ocular (colírios), nasal (sprays, gotas nasais) e vaginal (Brunton, 2012; Lourenço, A.R.N, 2013).

A administração de fármacos por via respiratória representa a forma de absorção medicamentosa mais rápida, quase que instantânea, comparada a todas as outras vias. Isto se 
deve ao grande enovelamento capilar em torno dos alvéolos pulmonares e à grande área de superfície pulmonar. A via inalatória é comumente utilizada para tratar doenças broncopulmonares e para administração de anestésicos gerais gasosos (Brunton, 2012; LIMA, M. F, 2008).

\section{CONCLUSÃO}

A farmacocinética constitui uma parte extremamente relevante da farmacologia na qual se estuda a forma com que o corpo processa os medicamentos, auxiliando na determinação de dose ideal e entendimento acerca das barreiras e velocidade que as substâncias precisam percorrer. É importante compreender a biodisponibilidade, isto é, aproveitamento de determinada substância pelo organismo, as vias de administração e o tempo gasto no seu local de ação, de maneira a assegurar um bom prognóstico para paciente, tratando-o sem que haja a ocorrência de efeitos indesejados.

\section{REFERÊNCIAS}

BRUNTON, L.L. As Bases Farmacológicas da Terapêutica de Goodman \& Gilman. 12 ed. Porto Alegre: Artmed, 2012. 2112 p.

DAVIS, C; HARRIS, S. R. USMLE Step 1 Lecture Notes 2017: Pharmacology. Kaplan Medical, 2017.

HERNANDEZ, E. M. M.; RODRIGUES, R. M. R.; TORRES, T. M.; ZUCOLOTO, A. D.;

OLIVEIRA, C. D. R.; EGITO, E. S. T. et al. Manual de toxicologia clínica: orientações para assistência e vigilância das intoxicações agudas. In Manual de toxicologia clínica:

orientações para assistência e vigilância das intoxicações agudas, p. 475-475, 2017.

KATZUNG, B. G.; TREVOR, A. J. Farmacologia Básica e Clínica-13. 13 ed. Porto Alegre: Artmed, 2017. 1216 p.

LE, J. Administração de medicamentos. Skaggs School of Pharmacy and Pharmaceutical Sciences, University of California San Diego. 2019.

LIMA, M. F. Formação em preparação e administração de medicamentos. Disponível em: http://www.farmaciamarques.com/Imgs/content/page_87/formacao\%20em\%20administra cao\%20de\%20medicamentos.pdf >. Acesso em 05 de Maio de 2021.

LOURENÇO, A.R.N. Administração Tópica De Fármacos - Das Restrições Aos Desafios. Disponível em: https://core.ac.uk/download/pdf/48580661.pdf. Acesso em 05 de julho de 2021. 
PEREIRA, D. G. Importância do metabolismo no planejamento de fármacos. Química nova, n. 30, p. 171-177, 2007.

ROSSATO, J.P.M. Avaliação da permeabilidade intestinal da furosemida e da furosemida complexada com hidroxipropil- $\hat{I}^{2}$-ciclodextrina por meio do modelo de perfusão in situ de passagem tripla em ratos. Tese de Doutorado. Universidade de São Paulo.

SOUZA, J. D.; FREITAS, Z. M. F.; STORPIRTIS, S. Modelos in vitro para determinação da absorção de fármacos e previsão da relação dissolução/absorção. Revista Brasileira de Ciências Farmacêuticas, n. 43, p. 515-527, 2007.

TRIPATHI, KD. Farmacologia Médica. 5 ed. Rio de Janeiro: Editora Guanabara Koogan, 2006. 196 p. 